\section{Collaborating against diabetes complications}

\section{By Michael J. Haas, Senior Writer}

Pfizer Inc.'s diabetes deal with the Sanford-Burnham Medical Research Institute could help reverse a trend that has seen the pharma arriving late-or not at all-to the party with new targets for the indication. Indeed, the goal of the three-year collaboration is to identify and validate new drug targets to prevent and treat insulin resistance and organ damage in obesity-related diabetes.

The only diabetes drug in Pfizer's product line is Actos pioglitazone, a thiazolidinedione that the company co-markets with Takeda Pharmaceutical Co. Ltd. Actos came off patent in 2008, and in 2011, the FDA required
"This collaboration with Sanford-Burnham is one example of how we approach the discovery and development of muchneeded therapies."

- Tim Rolph, Pfizer Inc.
The screening hits will serve as probes for validating the targets and for guiding the development of new therapies to prevent and treat insulin resistance and diabetes-induced organ damage, Vega said. Drugs that prevent or treat organ damage would have an advantage over the current standard of care for obesity-related diabetes.

Vega declined to disclose the financial terms of the deal or how ownership of any new IP generated by the collaboration will be handled.

"There is a clear need to translate innovative science into potential new medicines for people living with diabetes," said Tim Rolph, VP and head of the cardiovascular and metabolic diseases research unit at Pfizer. "This collaboration with Sanford-Burnham is one example of how we approach the discovery and development of much-needed therapies."

In 2011, Sanford-Burnham partnered with Pfizer to discover mechanisms and therapies for undisclosed diseases under the pharma's Global Centers for Therapeutic Innovation initiative.

Under that initiative, Pfizer provides its partners with funding for preclinical and clinical development programs and offers IP and ownership rights in return for options to license exclusive rights to drug candidates. Academic partners have access to Pfizer's antibody libraries and research and are eligible for milestones and royalties on advanced programs. ${ }^{2}$

Sanford-Burnham's push into the underpinnings of metabolic diseases dates to 2009. That year, the institute and Florida Hospital founded The Translational Research Institute for Metabolism and Diabetes (TRI), the warnings section of the drug's label to state that use of pioglitazone for more than a year may be associated with an increased risk of bladder cancer.

Pfizer's late-stage pipeline for the disease includes ertugliflozin, an oral sodium-glucose cotransporter 2 (SGLT2) inhibitor that is partnered with Merck \& Co. Inc. and is slated to start Phase III testing. The compound likely will be the fifth or sixth SGLT2 inhibitor to hit the market. ${ }^{1}$

However, Pfizer's earlier stage development efforts-the new deal with Sanford-Burnham and PF-05190457, a first-in-class ghrelin receptor (GHSR) antagonist in Phase I testing-look to reverse its late-to-theparty trend in diabetes.

Under the deal with Sanford-Burnham, Pfizer will use the institute's Conrad Prebys Center for Chemical Genomics to conduct high throughput screening for new targets using investigational compounds from Pfizer and a compound library from the NIH.

The partners plan to elucidate the mechanism of action for hit compounds to identify previously unknown disease targets involved in insulin resistance and damage to the heart, kidneys, retina and other organs caused by obesity-related diabetes, said Rick Vega, research assistant professor at Sanford-Burnham's Lake Nona campus in Orlando, Fla.

The collaborators also will draw on Sanford-Burnham's metabolomics capabilities and research facilities "to better understand the mechanism of action of the compounds that we identify and to make comparisons to known agents and reference compounds," he said. "These comparisons should help us prioritize the compounds of the highest interest." a joint venture aimed at identifying new markers that reflect subsets of heterogeneous metabolic diseases better than current markers.

TRI conducts clinical studies in patients with metabolic disease and healthy individuals, whereas a team at Sanford-Burnham's Lake Nona campus conducts preclinical research and provides metabolomics analyses. $^{3}$

In 2010, Sanford-Burnham and TRI signed a two-year deal with Takeda to support the clinical development of an undisclosed obesity compound from the pharma. The two institutes renewed the deal with Takeda in February and are open to forming partnerships with other drug companies, said Sanford-Burnham spokesperson Patrick Bartosch.

Haas, M.J. SciBX 6(33); doi:10.1038/scibx.2013.880

Published online Aug. 29, 2013

\section{REFERENCES}

1. Nallanathan, B. BioCentury 21(18), A9-A12; May 6, 2013

2. Cain, C. SciBX 3(46); doi:10.1038/scibx.2010.1371

3. Haas, M.J. SciBX 4(16); doi:10.1038/scibx.2011.446

\section{COMPANIES AND INSTITUTIONS MENTIONED}

Florida Hospital, Orlando, Fla.

Merck \& Co. Inc. (NYSE:MRK), Whitehouse Station, N.J.

Pfizer Inc. (NYSE:PFE), New York, N.Y.

Sanford-Burnham Medical Research Institute, La Jolla, Calif.

Takeda Pharmaceutical Co. Ltd. (Tokyo:4502), Osaka, Japan

The Translational Research Institute for Metabolism and

Diabetes, Winter Park, Fla. 